\title{
A NOVEL MULTI-SEGMENT PATH ANALYSIS-BASED TECHNIQUE FOR ACOUSTIC EMISSION SOURCE LOCALIZATION IN COMPLEX SOLID MEDIA
}

\author{
STEPHAN GOLLOB ${ }^{*}$, LASSAAD MHAMDI ${ }^{\dagger}$, GEORG K. KOCUR ${ }^{\ddagger}$, \\ THOMAS SCHUMACHER ${ }^{\dagger}$, AND THOMAS VOGEL ${ }^{*}$ \\ ${ }^{*}$ ETH Zurich \\ Zurich, Switzerland \\ e-mail: gollob@ibk.baug.ethz.ch \\ e-mail: vogel@ibk.baug.ethz.ch \\ ${ }^{\dagger}$ Portland State University \\ Portland, Oregon, USA \\ e-mail: lamhamdi@pdx.edu \\ e-mail: thomas.schumacher@pdx.edu \\ ${ }^{\ddagger}$ Halfen $\mathrm{GmbH}$ \\ Langenfeld, Germany \\ e-mail: georg.kocur@halfen.de
}

Key words: Acoustic Emission, Wave Propagation, Pencil-Lead Breaks, Multi-Segment Wave Propagation Paths, Source Location, FastWay

\begin{abstract}
FastWay is a novel method for source localization of acoustic emissions (AE) in complex solid media. It uses the fastest, rather than the shortest wave path between the AE source and the recording sensors. In this paper we investigate the potential of this method for acoustic emission source localization in concrete structures. To consider the influence on the wave propagation of both the concrete heterogeneity and the possible cracks present in the tested specimen, a heterogeneous velocity model was selected and a multi-segment path analysis based on this model was performed. After validating the model numerically using simulated AE sources, laboratory experiments were conducted on a small concrete beam $(152 \mathrm{~mm} \times 152 \mathrm{~mm} \times 533 \mathrm{~mm})$ with a predefined notch cut to serve as a material discontinuity (crack). Artificial AE sources using pencil-lead breaks were applied on a $25.4 \mathrm{~mm} \times 25.4$ point grid mapped on the surface of the beam. To evaluate the performance of FastWay, a set of sources randomly selected were picked and localization results using both FastWay and Geiger's method compared. The results obtained show that FastWay performs more reliably and accurately than Geiger's algorithm even in the presence of cracks and air inclusions. No major influence of these two factors was seen on the localization results. The influence considered the most crucial, however, is of the velocity model which strongly depends on the complex internal structure of the tested specimen.
\end{abstract}

\section{INTRODUCTION}

Acoustic Emission (AE) is a powerful technique with worldwide use in nondestructive tes- ting (NDT) and monitoring applications. Like many other NDT methods, AE has a basic concept, a distinctive terminology and different 
characteristic instrumentation, parameters and fundamentals. The general concept of the $\mathrm{AE}$ states that a structure, a component or a piece of machinery subjected to an external stimulus (change in load, temperature pressure, etc) will undergo internal changes in form of stress redistributions that result in sudden release of energy within the material medium. The energy released radiates outward in form of elastic transient waves [1] that propagate through the medium toward the surface of the specimen being tested. By mounting a network of uniaxial sensors on the surface of this specimen, one can listen to audible noise, record acoustic sounds in form of electric signals and perform different types of analyses in order to evaluate the material condition and detect possible flaws and defects [7]. By analyzing the data recorded by the sensors, fracture processes can be monitored over time and condition changes of the observed member inferred. In case of cracked and/or reinforced concrete, the elastic wave will mostly not propagate along a straight path, as it is presumed by common source location estimations methods (e.g. Geiger's method). For instance, in the presence of cracks, generally filled with air, the wave has to change its travelling path since no elastic energy transmits through air. Also, the velocity of the propagating wave is usually variable due to the heterogeneity nature of concrete. This velocity variation considerably affects the wave behavior and should be therefore taken into account. In order to consider the influence on the wave propagation of both the concrete heterogeneity and the possible cracks present in the tested specimen, a heterogeneous velocity model was selected and a multi-segment path analysis based on this model was incorporated into a novel source location estimation method, called FastWay. To evaluate the performance of FastWay, a set of sources randomly selected were picked and localization results using both
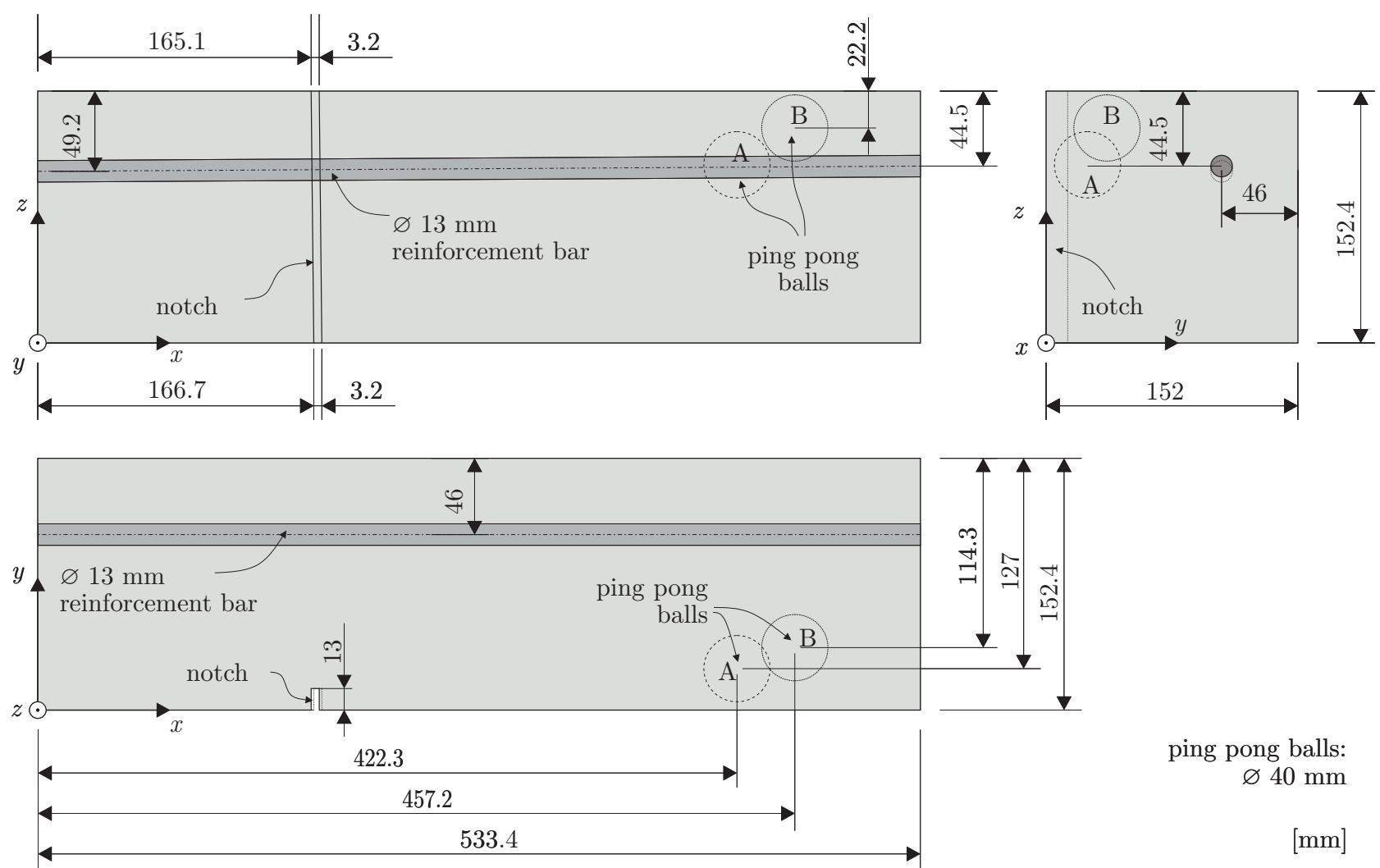

ping pong balls: $\varnothing 40 \mathrm{~mm}$

[mm]

Figure 1: Visualization of the reinforced, notched concrete specimen in three sections. The positions of the ping pong balls, the notch and the reinforcement is marked and dimensioned. 
FastWay and Geiger's method compared.

\section{EXPERIMENTAL SETUP}

The experimental work was performed in the laboratory at the University of Delaware where a small concrete beam of dimensions $(152 \mathrm{~mm} \times 152 \mathrm{~mm} \times 533 \mathrm{~mm})$ was cast, cured and tested. A $\varnothing 13 \mathrm{~mm}$ reinforcing bar as well as two identical ping pong balls with a diameter of $40 \mathrm{~mm}$ each were placed in the beam during casting. This aims at producing a very heterogeneous medium with different components (concrete, steel, air). The positions of the ping pong balls as well as of the steel reinforcing bar are shown in Figure 1. A notch was also cut in the beam to serve as a crack and further complicate the geometry of the propagating medium. The beam was then mapped with a $25.4 \mathrm{~mm} \times 25.4 \mathrm{~mm}$ point grid placed on the whole surface and pencil-lead breaks were used on each grid point to generate acoustic emission sources. Twelve piezoelectric sensors were mounted on the surface of the beam to record the AE signals generated by the pencillead breaks.

\section{LOCALIZATION METHODS}

Two arrival-time-based methods were used to estimate the locations of 26 sources picked randomly on the grid. Two input values are required for both methods; (1) the wave arrival times to the sensors and (2) the velocity model assigned to the specimen. While picking the arrival times can be, in many cases, a challenging problem as the wave onset time cannot be picked with $100 \%$ accuracy, several onset time picking techniques are available in the literature (e.g. threshold technique, AIC-based technique [8], etc.) that can be used reliably in time arrival picking problems. The arrival time picking is not the main focus of this document. The velocity model is, however, of main concern in this study. In all what follows, the p-wave velocity in air was assumed to be zero, the p-wave velocity in steel was assumed to be $c_{p}=5900 \mathrm{~m} / \mathrm{s}$ (value taken from [6]) and the p-wave velocity in concrete was determined experimentally. The location of the sensors and the source are known a priori. This information can be used to determine an average wave propagation velocity. If all sensors that might not be reached by a wave following a direct path (approximately straight) are excluded, the average velocity obtained should be identical to the p-wave velocity in concrete. Hence, sensors that might be reached first by an elastic wave propagating through the reinforcing bar, as well as all sensors separated by air (notch or ping pong balls) from the source are excluded. Figure 2 shows the result of the velocity identification strategy performed for source P92 which is located on the plane $z=0$ of the specimen. The signals visualized with dotted lines are excluded for one of the reasons mentioned above. The regression line displays the average $\mathrm{p}$-wave velocity obtained for concrete $\left(c_{p}=4404 \mathrm{~m} / \mathrm{s}\right)$. Applying the same identification strategy for different sources leads to an average $\mathrm{p}$-wave velocity of $c_{p} \approx 4400 \mathrm{~m} / \mathrm{s}$.

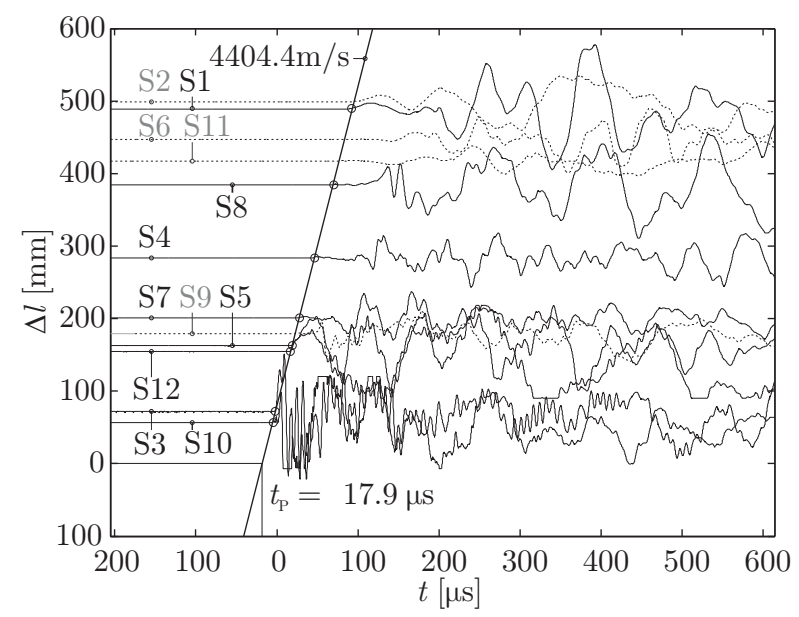

Figure 2: AE signals recorded by the twelve sensors mounted on the beam and sorted with respect to their distance $\Delta l$ to the location of the known source. Signals excluded from the velocity identification are plotted as dotted lines. The arrival time of the wave to the sensor is marked with a $\circ$. The slope of the regression line corresponds to the average velocity $c_{p}$.

\subsection{GEIGER'S METHOD}

The most common method for AE source localization is the Geiger's method [3]. This me- 
thod assumes straight wave propagation paths and a constant wave propagation velocity. In case of homogeneous uncracked specimens (e.g. a steel pressure vessel with no crack), these assumptions are valid, and the Geiger's method generates accurate source location estimations. The constant global wave velocity implemented for the whole specimen, including the notch, the ping pong balls, and the reinforcing bar is $c_{p}=4400 \mathrm{~m}$.

\subsection{FASTWAY}

The source location method of FastWay relies on the fastest, rather than the shortest (the straight) wave propagation path between the sensors and the AE source. The fastest wave propagation path is obtained with a modified Dijkstra algorithm [2]. A numerically discretized model of the specimen is needed as input [5]. The location of the reinforcement, the existing air inclusions (like the ping pong balls), the notches and cracks have to be known a priori in order to provide an accurate numerical model of the specimen. The $\mathrm{p}$-wave velocities assigned to the three different materials included in this numerical model are $c_{p}=0 \mathrm{~m} / \mathrm{s}$ for air, $c_{p}=5900 \mathrm{~m} / \mathrm{s}$ for steel and $c_{p}=4400 \mathrm{~m} / \mathrm{s}$ for concrete as mentioned earlier. Since the reinforcing bar behaves as a wave guide and the velocity of the guided wave is usually slower than the $\mathrm{p}$-wave velocity in steel $\left(c_{p}=5900 \mathrm{~m} / \mathrm{s}\right)$, the wave velocity implemented for steel in FastWay was set to $c_{p}=5150 \mathrm{~m} / \mathrm{s}$. The FastWay algorithm does not compute a source location directly. Instead, based on the velocity model and the arrival times of the wave at all the sensors used, FastWay investigates which voxels (three-dimensional pixels) most likely host the source [4]. Based on the beforehand computed fastest wave path and the picked arrival time at one sensor a theoretical source time for a source located in the center of each voxel can be determined. A source located at the center of one voxel combined with the corresponding deter-
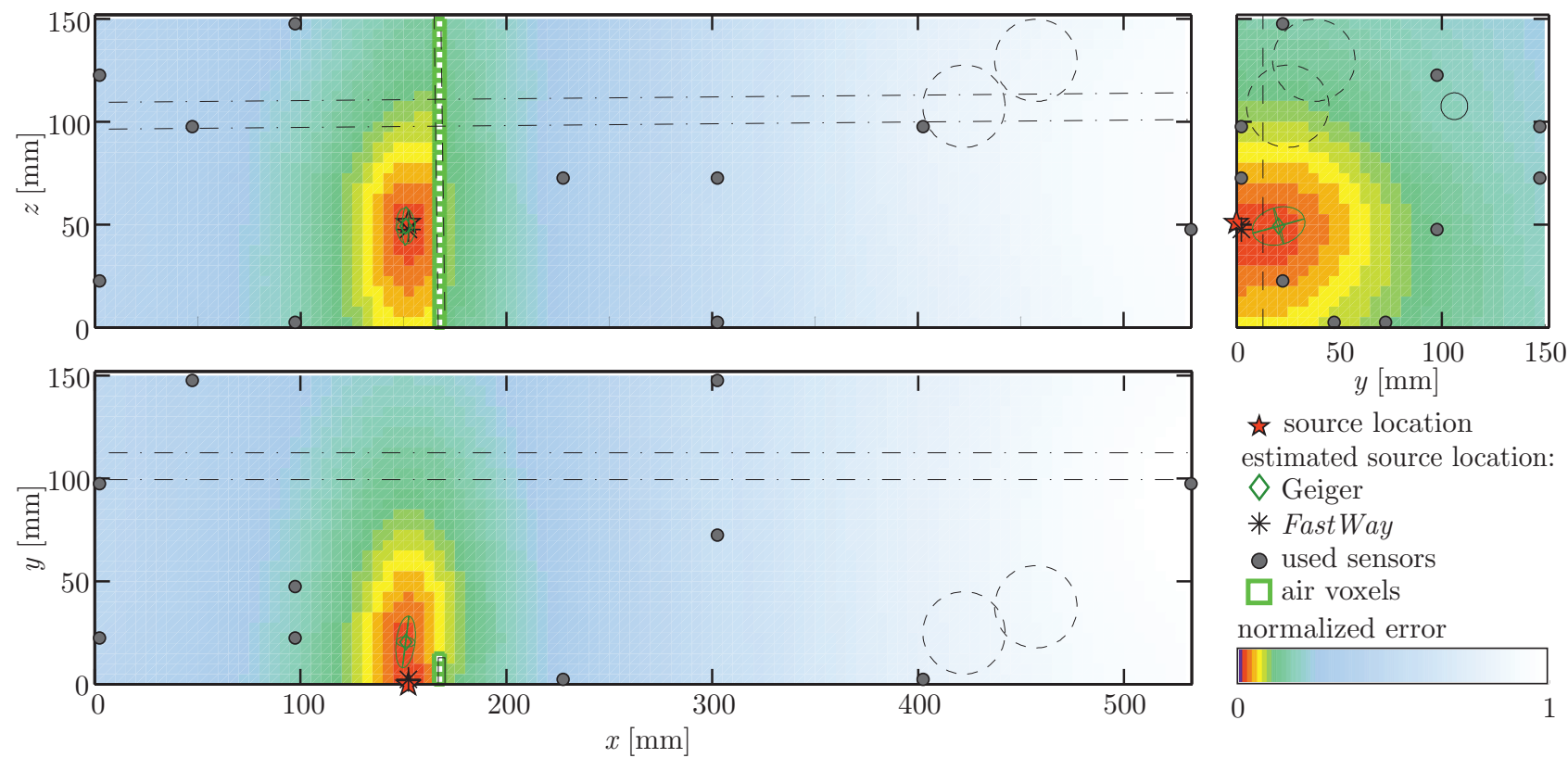

须 source location estimated source location: $\diamond$ Geiger

* FastWay

- used sensors

air voxels

normalized error

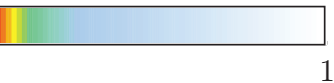

Figure 3: Visualization of the results of estimating the location of source P27 located on plane $y=0 \mathrm{~mm}$. Sections including visualizations of $\mathbf{E}_{\text {source }}$, error ellipsoids for the Geiger's method, locations of the sensors, estimated source locations and actual source location are marked; cross sections positioned at location of the source estimated with FastWay. The position of the actual ping pong balls, the notch as well as the reinforcing bar are drawn in the sections. The voxels representing air in the numerical discretized model are highlighted with green borders. 
mined source time would result in the same arrival time of the wave at the sensor as the picked on. The determined source times of each voxel can be combined in a matrix of the same size as the discretized numerical model of the specimen. This process is repeated for each sensor separately. In theory, one entry in each matrix is identical with the corresponding entry in every other source time matrix, representing the source time of the investigated AE event. The matrix entry corresponds to one voxel of the numerical model. This voxel most likely hosts the source. The accuracy of FastWay strongly depends

on the accuracy of the implemented numerical model of the specimen. Moreover, the resolution of the implemented numerical model has a considerable influence on the precision of the estimated source location, since only the voxel that most likely hosts the source but not the exact location of the source within the voxel can be determined.

\section{RESULTS}

The results of the Geiger's source localization algorithm are the coordinates of an estimated source location. Error ellipsoids are calcula-
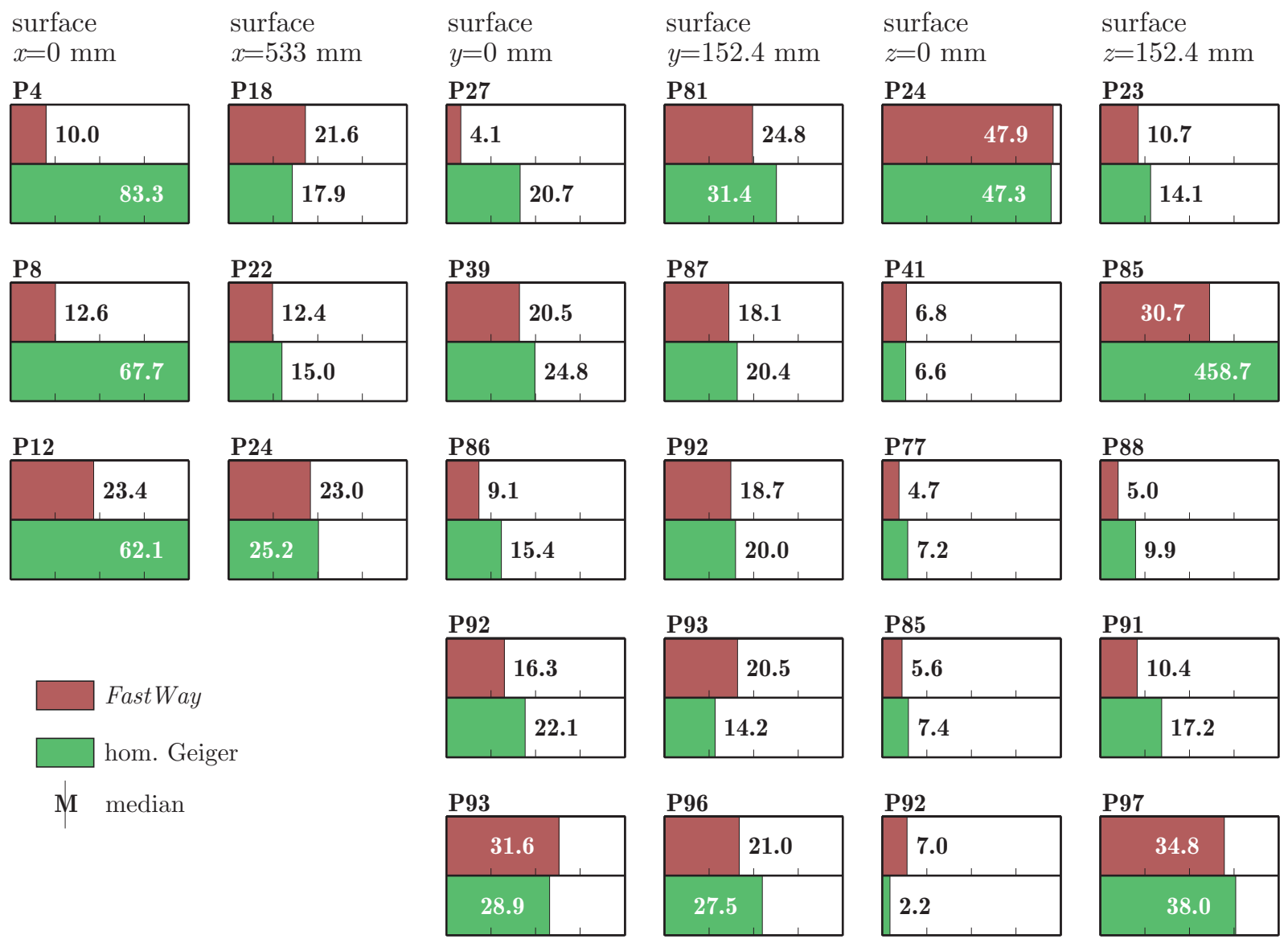

mean value of all results of each surface
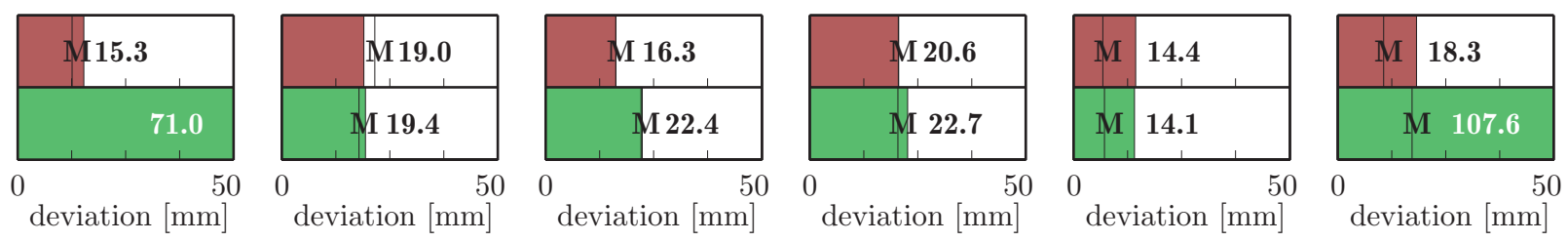

Figure 4: Display of the deviation between the estimated and the actual source locations. In the last row the mean (colored bar) and the median of the deviation computed for each surface separately are shown. 
ted to visualize the estimated accuracy of the solution obtained. The result of FastWay is a normalized error matrix, $\mathbf{E}_{\text {source}}$. The values of $\mathbf{E}_{\text {source }}$ represent the normalized deviations between the theoretical source times determined for each sensor and range from 0 to 1 . The lowest value corresponds to the voxel that is most likely hosting the source. These results can be visualized as in Figure 3. Both methods provide satisfactory location results. The errors between the estimated source locations and the exact real location is less than $25 \mathrm{~mm}$. However, the exact location is located outside of the error ellipsoid computed for the result provided by Geiger's method. The visualized result computed with FastWay is even more accurate (error of $4.1 \mathrm{~mm}$ ), than the result computed with the Geiger's method (error of $20.7 \mathrm{~mm}$ ). The colored plot indicates a certain area where the source is most probably located at. It is clearly visible that the source must be located at the surface $y=0$. It has to be mentioned that not all results provided by FastWay and Geiger's method are as satisfying as the one shown in Figure 3. FastWay was not able to locate four sources with an error of less than $25 \mathrm{~mm}$. This particular result is considered to be a rouge result. The maximum error between the actual source location and the location estimated with FastWay is $47.9 \mathrm{~mm}$. Geiger's method was not able to locate ten sources with an error of less than $25 \mathrm{~mm}$. The maximum error between the actual source location and the location estimated with this method is $458.7 \mathrm{~mm}$. The second biggest error between the actual source location and the location estimated with the Geiger's method is $83.3 \mathrm{~mm}$. A comparison of the deviation between the source locations estimated with both methods (FastWay and Geiger's) and the actual source location is shown in Figure 4

\section{CONCLUSIONS}

FastWay was able to provide more accurate source location estimations than Geiger's method in most of the cases studied (twenty of twenty-six). Geiger's method only estimated six out of twenty-six sources more accurately than FastWay. The sources located in the vicinity of the ping pong balls could not be estimated well using both methods (e.g. P24 located at $(x=533.4 / y=127 / z=127)$ $[\mathrm{mm}])$. To conclude, the evaluation of the experimental data using the two aforementioned arrival-time-based source location methods have demonstrated that FastWay is capable of processing experimental data and is able to provide more satisfying results. The average error between the estimated source location and the actual one was equal to $17.2 \mathrm{~mm}$ (median: $17.4 \mathrm{~mm}$ ) for the case of FastWay, and $42.6 \mathrm{~mm}$ (median: $20.6 \mathrm{~mm}$ ) for Geiger's method. In the majority of the investigated sources FastWay provided more accurate results.

\section{REFERENCES}

[1] J. D. Achenbach. Wave Propagation in Elastic Solids (Applied Mathematics and Mechanics). North Holland Publishing Company, Amsterdam, 1987.

[2] E. W. Dijkstra. A Note on Two Problems in Connexion with Graphs. Numerische Mathematik 1, pages 269-271, 1959.

[3] Moachen Ge. Analysis of source location algorithms, Part II: Iterative methodes. Journal of Acoustic Emission, 21:29-51, 2003.

[4] Stephan Gollob, Georg Karl Kocur, and Thomas Vogel. Localization of Acoustic Emissions in a numerically simulated Tshaped Concrete Beam. In Proceedings WCAE-2015, 2015.

[5] Stephan Gollob and Thomas Vogel. Localization of Acoustic Emission in Reinforced Concrete using a Heterogeneous Velocity Model and Multilinear Wave Propagation Paths. In Proceedings - Fib Symposium 2015 - Copenhagen, 2015.

[6] Georg Karl Kocur, Thomas Vogel, and Erik H. Saenger. Crack localization in a double-punched concrete cuboid with time reverse modeling of acoustic emissions. International Journal of Fracture, 171:1-10, 2011.

[7] Lassaad Mhamdi. Seismology-based Ap- 
proaches for the Quantitative Acoustic Emission Monitoring of Concrete Structures. $\mathrm{PhD}$ thesis, University of Delaware, 2015.

[8] B. Schechinger and T. Vogel. Acoustic emission for monitoring a reinforced concrete beam subject to four-pointbending. Construction and Building Materials, 21(3):483-490, 2007. 